\title{
Transcription factor PU.1 is involved in the progression of glioma
}

\author{
YUANZHI XU ${ }^{1 *}$, SONG GU ${ }^{2 *}$, YUNKE BI ${ }^{1}$, XIANGQIAN QI ${ }^{1}$, YUJIN YAN ${ }^{3}$ and MEIQING LOU ${ }^{1}$ \\ ${ }^{1}$ Department of Neurosurgery, Shanghai First People's Hospital, Shanghai Jiao Tong University, School of \\ Medicine, Shanghai 200080; ${ }^{2}$ Department of Neurosurgery, The First Affiliated Hospital of \\ Anhui Medical University, Hefei, Anhui 230022; ${ }^{3}$ Department of Neurosurgery, The Affiliated Hospital of \\ Medical School of Ningbo University, Ningbo, Zhejiang 315020, P.R. China
}

Received June 20, 2017; Accepted January 3, 2018

DOI: $10.3892 / \mathrm{ol} .2018 .7766$

\begin{abstract}
Glioma is a severe disease of the central nervous system. Although previous studies have identified the important role of the immune response in association with tumor intervention, it is still unknown whether PU.1, a transcription factor known for its role in myeloid differentiation and immune responses, is involved in the progression of glioma. In the present study, we found a significant increase in SPI, the gene that encodes PU.1, in samples from patients with glioma. Through genotype-phenotype association analysis several candidate factors that may mediate the role of PU.1 in glioma were identified. To further validate the association between PU.1 and glioma we found that the expression of BTK, a potential target of PU.1, was also upregulated in patients with glioma. We also demonstrated that various biological pathways could be involved in PU.1-associated glioma by analyzing these potential targets in the Reactome database. These results provide evidence that PU.1 could serve a role in the progress of glioma through its transcriptional targets in multiple signaling pathways. Therefore, in addition to its role in hematopoietic linage development and leukemia, PU.1 appears to be involved in the regulation of glioma and potentially in other malignant cancers.
\end{abstract}

\section{Introduction}

Cancer is one of the most malignant diseases worldwide. Numerous signaling pathways have been identified involved in

Correspondence to: Dr Yujin Yan, Department of Neurosurgery, The Affiliated Hospital of Medical School of Ningbo University, 247 People's Road, Ningbo, Zhejiang 315020, P.R. China E-mail: 1376090092@163.com

Dr Meiqing Lou, Department of Neurosurgery, Shanghai First People's Hospital, Shanghai Jiao Tong University, School of Medicine, 100 Haining Road, Shanghai 200080, P.R. China

E-mail: loumq68128@hotmail.com

*Contributed equally

Key words: PU.1, glioma, BTK, transcriptional targets the development of severe cancers. Abnormal transcriptional programs have been reported playing important roles in the generation and development of malignant carcinoma (1). Expression profiling using cDNA microarray on primary samples extracted from patients, as well as established mouse models, has revealed hundreds of transcriptional factors that are dysregulated in various types of cancers, including PU.1 (2).

PU.1 is a hematopoietic transcription factor, known for its role in myeloid development (3). PU.1 depletion is lethal for animal during embryonic development due to hematopoietic failure, with abnormal lymphoid and myeloid cell lineages (3-5). It has also been implicated in tumor progression, especially in leukemia (2). Strong PU.1 expression were shown associated with longer survival in follicular lymphoma (6). Deletion and downregulation of PU.1 were also found in human acute myeloid leukemia (AML) and indolent types of lymphomas $(7,8)$. On the contrary, surprising results were reported in a recent study by Zhou et al showing that PU.1 is required and works as an essential regulator for the development of mixed lineage leukemia (MLL) (9), one of the aggressive forms of AMLs with poor prognosis $(10,11)$. The molecular mechanism for PU.1 in tumor progression has not been fully identified. Some study indicated that PU.1 could contribute to leukemia through transcriptional activation of leukemia fusion proteins (9). It is also reported to promote cytokine production as well as monocytes activation (12), indicating PU.1 could affect tumor severity through immune responses. Although PU.1 play contradictory roles in different types of leukemia, whether PU.1 is involved in other types of cancers is not known.

Glioma is a devastating brain cancer (13). Patients diagnosed with glioblastoma multiforme (GBM) have low survival rate and high morbidity, despite all kinds of medical treatments (14). Although tumors in the brain suffer many restrictions for clinical therapy, such as the blood brain barrier, similar to other solid tumors, the immune cells, i.e., microglia in the brain, are attracted to the GBM tissues and support tumor progression (15-19). Thus recently studies have been done focusing on glioma-associated immune cells for disease intervention (20,21). Although PU.1 is important for immune cells in the circulatory system, its role in the central nervous system, especially in glioma, is unknown.

Here we examined the clinical relevance of the PU.1 in patients with different kinds of gliomas, and found that the 
expression of genes coding PU.1 is significantly increased in glioma patient samples. We also aim to explore potential factors involved in PU.1 signaling and glioma. Using the online available tool to search for the genotype-phenotype associations between PU.1 and glioma, we found that several genes could be the targets of PU.1 in mediating tumor severity. Our results provide initiative evidence that PU.1 could contributes to the progress of glioma, probably via its targeting gene signatures.

\section{Materials and methods}

GEO datasets and statistical analysis. Publicly available gene-expression datasets of glioma patients were downloaded from NCBI-GEO with accession numbers GSE4290 and GSE15824 (http://www.ncbi.nlm.nih.gov/geo/). Statistical analysis for relative gene-expression data were performed using DESeq2 version:v1.4.5. The P-values were calculated using the Benjamini and Hochberg method in the $\mathrm{R}$ DESeq2 package.

Immunostaining. Biospecimens were obtained from patients from the Shanghai First People's Hospital, according to their surgical pathology reports and clinical records. The study was approved by the Ethics Committee of the Shanghai First People's Hospigal, Shanghai Jiaotong University. All subjects agreed to donate their tissue for this study. Tissues were fixed in formalin. Slicing and immunohistochemistry were performed according to standard protocol in a previous study (22). Antibodies used are: PU.1, 9G7 Rb IgG, 1:100, Novex; CD68, FA11 Rt IgG, 1:200, Serotec.

Genotype-phenotype association analysis. Analysis was performed using an online system to search for genotype-phenotype associations (http://literome.azurewebsites. net/). Key words used to search for genotype-phenotype associations are 'SPI1' and 'glioma'.

Target gene identification. Online available database were applied to identify transcription targets of PU.1 (https://cb.utdallas.edu/cgi-bin/TRED/tred.cgi? process=searchTFGeneForm). 90 genes were identified as potential targets of PU.1.

Biological pathway analysis. Biological pathway analysis was performed using an online available tool, the Reactome database (http://www.reactome.org/). Multiple biological pathways in Homo sapiens were showed linked to the network containing genes of interest.

\section{Results}

To examine the clinical relevance of PU.1 expression in glioma patients, we performed analysis on SPIl (gene that encode PU.1) expression using publicly available RNA-sequencing array data. Each dataset can be classified as four groups: Non-tumor sample, astrocytoma patient sample, glioblastoma patient sample and oligodendrioglioma patient sample. Analysis was performed as control vs. disease conditions. Non-tumor samples were shared for comparisons in each

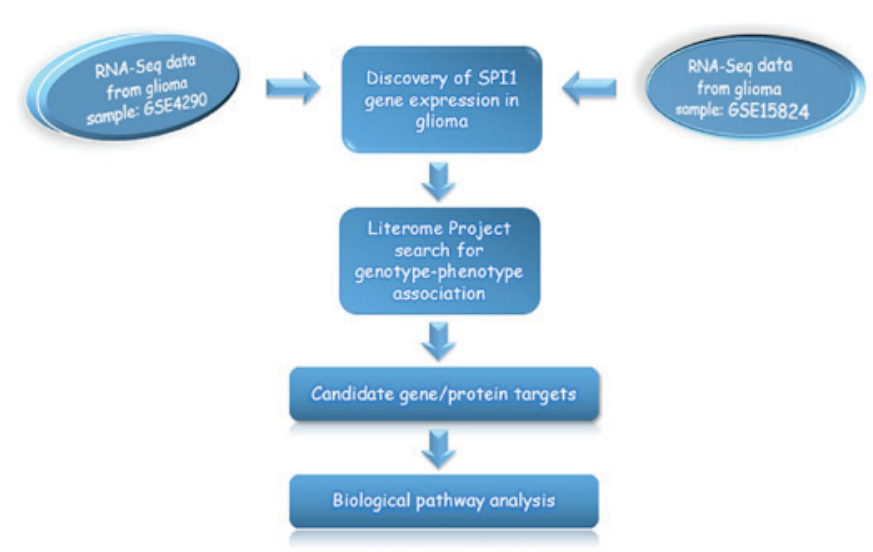

Figure 1. Illustrative flowchart showing the research procedure.

dataset. Fig. 1 detailed the analysis strategy included in this study. The position of gene SPII in genome of Homo sapiens was shown in Fig. 2A. As shown in Fig. 2B, we found significant increases of SPII mRNA expression in several kinds of glioma samples, while there is trend of upregulated SPII expression in all glioma samples, including astrocytoma, glioblastoma and oligodendrioglioma.

We next explored such correlation in another public database, the REMBRANDT database (http://www.betastasis. com/glioma/rembrandt/). We found that SPII expression levels were significantly increased in patients with glioblastoma and astrocytoma (Fig. 2C). These results suggest clear positive correlation between SPI1 expression and glioma, especially glioblastoma and astrocytoma.

To further demonstrate the relevance between PU.1 expression and glioma malignancy, we tested PU.1 protein expression in patient brain tissues. We found that PU.1 was highly expressed in glioma patients, but not in non-glioma patients (Fig. 3). CD68 was stained as marker of macrophage. Some of the PU.1 positive cells are colocalized with the CD68 ${ }^{+}$cells.

We also searched TCGA database for further evidence. Three genomic mutations have been identified in two glioma patients at the SPI1 locus (23). These mutations include: Chr11:g.47378343G>A, chr11:g.47359851A>T, chr11:g.47375730C $>$ A. Interestingly, we also noticed a negative correlation between SPI1 expression and patient survival rate (Kaplan-Meier estimator, $\mathrm{P}=0.0035$ ) in the REMBRANDT database, further demonstrating the involvement of PU.1 in glioma malignance.

Next we are interested in what are the potential mechanisms that PU.1 is involved in glioma. We applied analysis using an online system to search for genotype-phenotype associations (http://literome.azurewebsites.net/) $(24,25)$. The basic principle is to extract genomic and phenotypic knowledge from PubMed articles thus providing possible signaling pathways for one gene's function. Using this online available tool, we found associations between SPII and glioma via 8 different genes, including BTK, ERVK-6, FLI1, FURIN, GRAP2, IL1B, MYLIP and SERPINB1. We further examined the viability of these associations through literature studying, and identified that several genes could be the potential targets of PU.1 in mediating glioma severity. BTK 
A

A

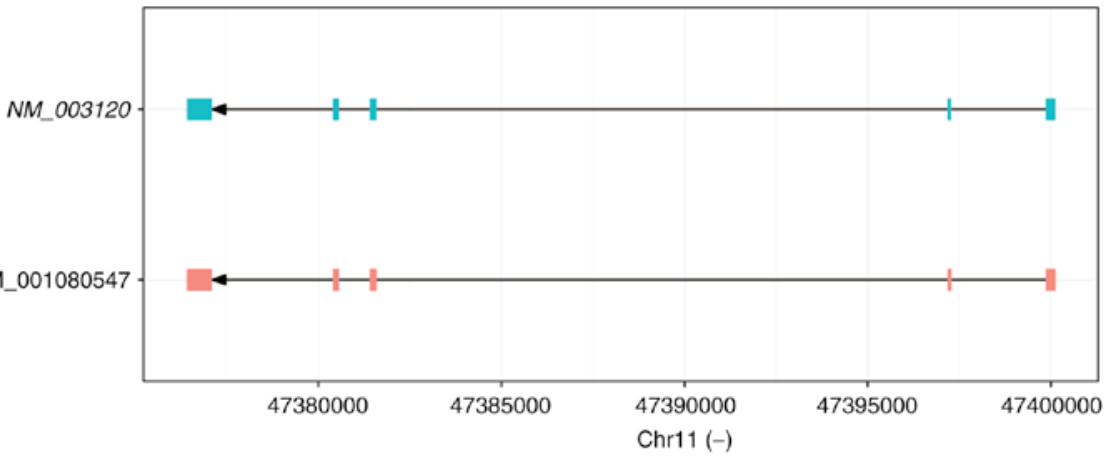

B

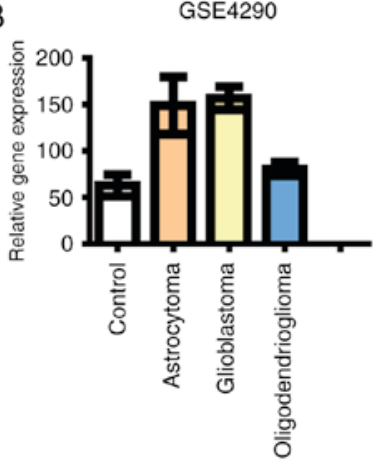

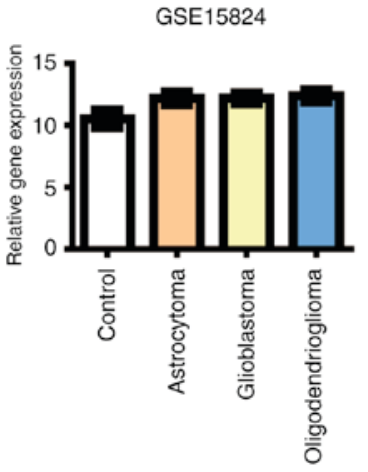

C

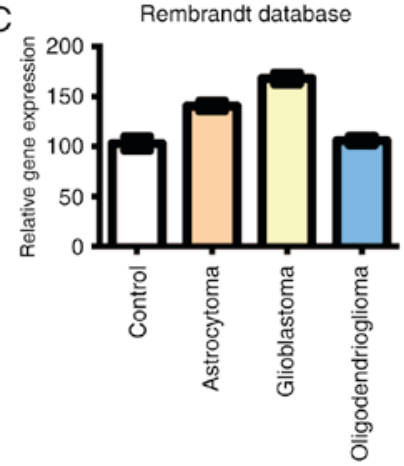

Figure 2. Relative expression of SPI1 was increased in glioma patient. (A) Gene locus for SPI1 transcription at the genome. Transcript in italic was analyzed in the study. Arrows, direction of transcription. Box, exons in indicated genes. Each line indicated one transcript for the corresponding gene. (B and C) Fold changes of gene expression levels in three types of glioma samples. Bar graphs showed average fold changes in astrocytoma, glioblastoma and oligodendrioglioma samples. Database used was as indicated.
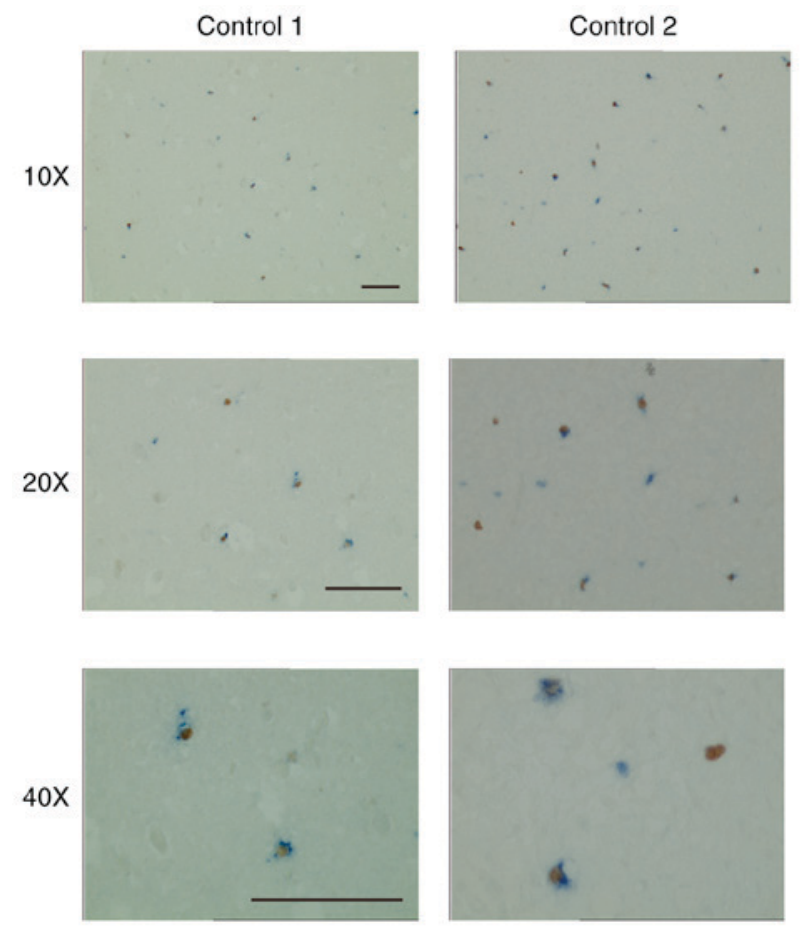
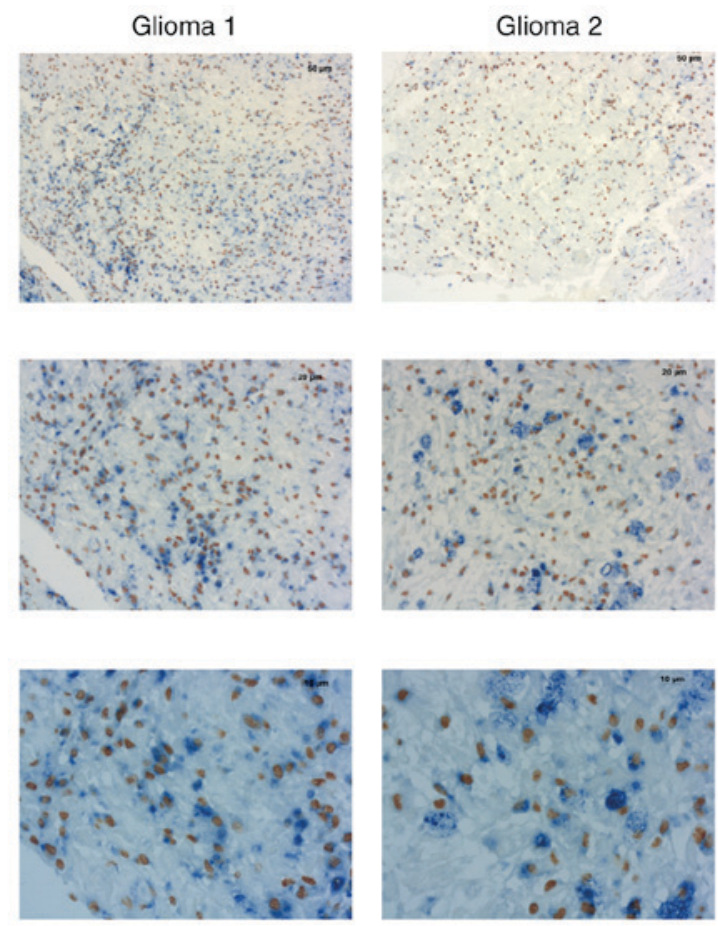

Figure 3. Images of brain tissues from normal and glioma patients. Brown, PU.1 staining; blue, CD68 staining. Scale bars, $100 \mu \mathrm{m}$.

is the Bruton's agammaglobulinemia tyrosine kinase, known for its role in X-linked agammaglobulinemia. It is reported that BTX gene contains PU.1 binding sites at its promoter region. In PU.1 $1^{-/}$mice, BTK expression was significantly reduced (26). Another important potential regulator is FLI1, a transcription factor in the ETS family. FLI1 transcription was regulated under SPI1 transfection (27). PU.1 was also reported binding to the promoter of IL1B to activate its expression (12). These three candidates were all found involved in the progress of glioma (28-30). 


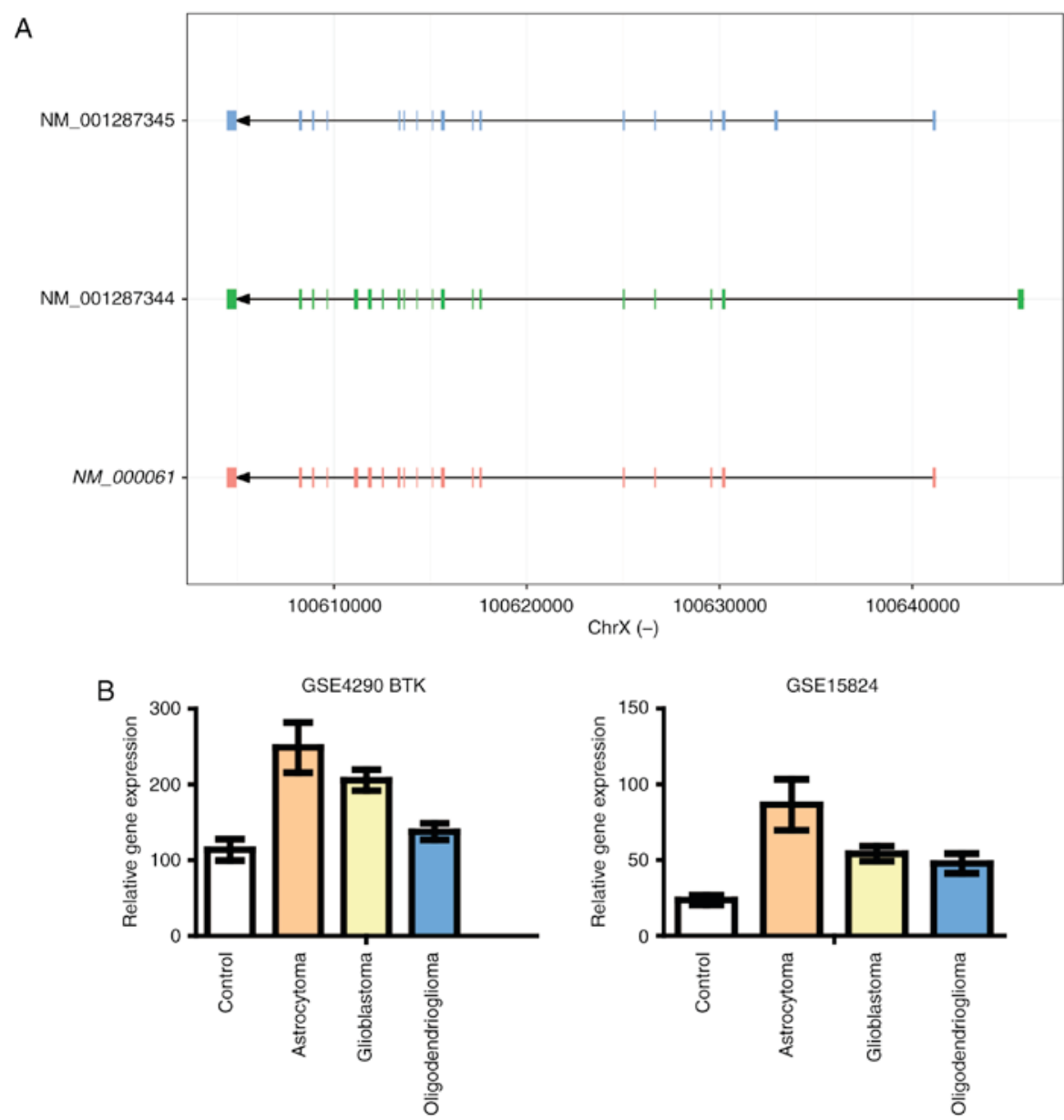

Figure 4. Relative expression of BTK was increased in glioma patient. (A) Gene locus for BTK transcription at the genome. Transcript in italic was analyzed in the study. Arrows, direction of transcription. Box, exons in indicated genes. Each line indicated one transcript for the corresponding gene. (B) Fold changes of gene expression levels in three types of glioma samples. Database used was as indicated. BTK, Bruton's agammaglobulinemia tyrosine kinase.

We analyzed the expression changes of the three predicted genes in glioma RNA array data used for analysis of SPII expression. As shown in Fig. 4, we found significant increases of $B T K$ mRNA expression in both glioma datasets. On the other hand, FLII mRNA level was increased in GSE4290 dataset, while decreased in the GSE15824 dataset (Fig. 5B). In terms of the expression level of $I L 1 B$, it was decreased in the GSE4290 dataset, while increased in the GSE15824 dataset (Fig. 6). We plotted the fold changes of these genes as bar graphs, as shown in Figs. 4-6, the expression pattern of $B T K$ is more comparable to that of the gene SPII. These results suggest that BTK is more probably an important factor involved in the role of PU.1 in glioma progression.

Although only three genes were predicted involved in the regulation of glioma by PU.1, PU.1 could target a number of genes via transcriptional regulation. Taking advantage of an online available tool, we identified 90 potential transcription targets of PU.1 (https://cb.utdallas.edu/cgi-bin/TRED/tred. cgi?process $=$ searchTFGeneForm). We analyzed the expression levels of these genes in glioma samples (data not shown). The results showed that genes, such as LTF, FSF1, CD163 and MMP2, were consistently upregulated in glioma tissues. Although there is no direct link for these factors and brain tumor, indirect mechanisms/signaling pathways could be involved in the role of PU.1 in glioma. In summary, our results indicate that PU.1 could contribute to the progress of glioma, probably via its targeting gene signatures.

We next searched for biological pathways linked to the PU.1-associated genes in glioma using Reactome database $(31,32)$. Three nodes were identified representing PU.1-targeting pathways in glioma, including immune system, signal transduction, and disease. Meanwhile, multiple biological pathways were found associated with thus process, including the immune system, signal transduction, disease, gene expression, and metabolism of proteins.

In conclusion, our study identified significant increase of PU.1 expression in human glioma patients. Through genotype-phenotype association test and biological interaction network building, our results indicate that PU.1 could affect the development of glioma by targeting various genes in different signaling pathways.

\section{Discussion}

Glioma is a kind of severe cancer starts in the brain or spinal cord with low survival rate and high morbidity $(13,14)$. Although previous studies have implicated a lot of signaling pathways involved in the progress of glioma, until now there is no cure for the severe disease. Thus, it is urgent to identify more 


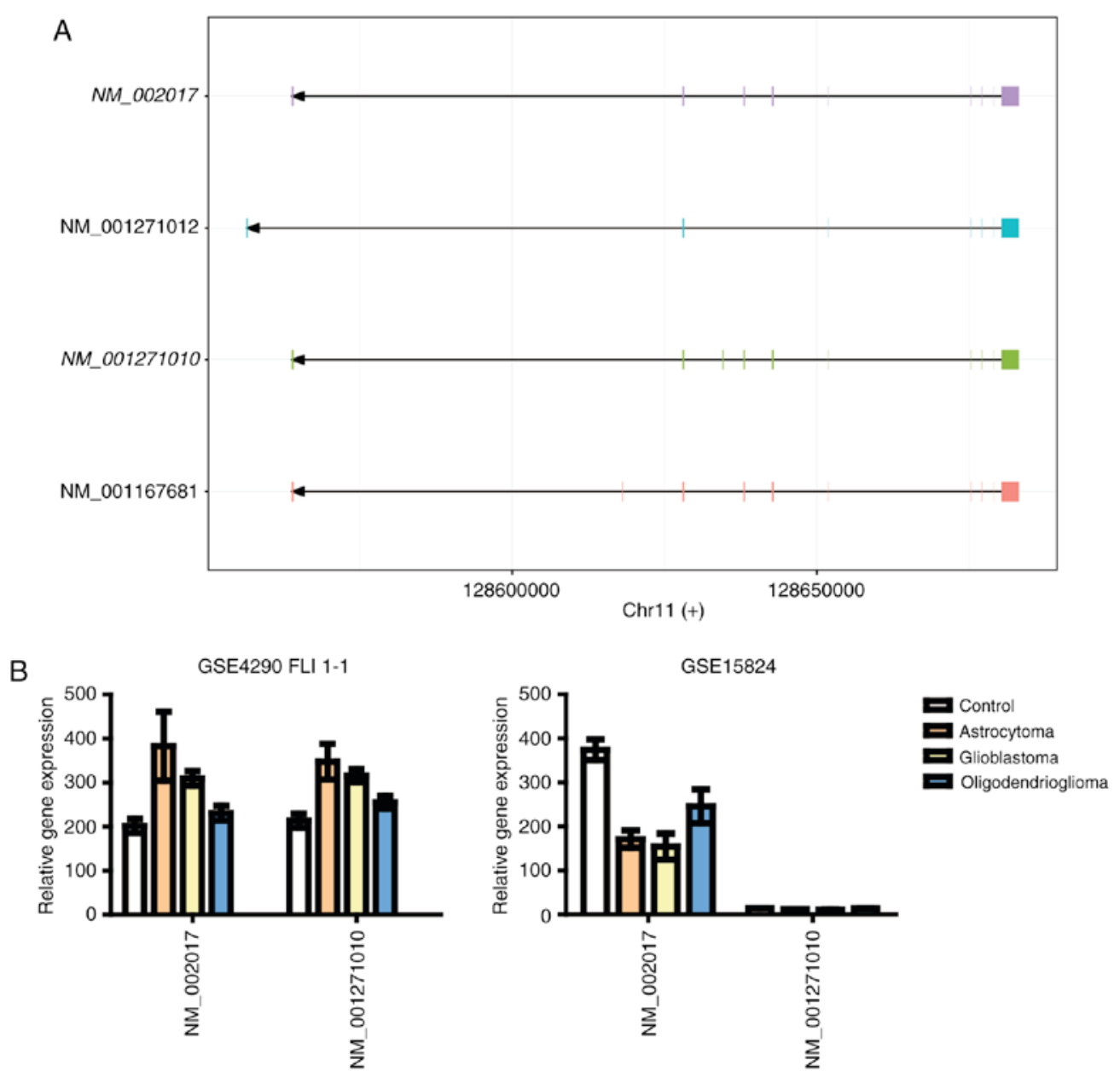

Figure 5. Relative expression of FLI1 was increased in glioma patient. (A) Gene locus for FLI1 transcription at the genome. Transcripts in italic were analyzed in the study. Arrows, direction of transcription. Box, exons in indicated genes. Each line indicated one transcript for the corresponding gene. (B) Fold changes of gene expression levels in three types of glioma samples. FLI1, friend leukemia integration 1 transcription factor.

changes in glioma condition for medical treatment. However, it is still unknown whether PU.1, a transcription factor known for its role in myeloid differentiation and immune responses, is involved in the progress of glioma.

In this present study, we found significant increase of SPII, the gene coding transcription factor PU.1, in glioma patient samples through analysis of RNA array dataset. We hypothesized that PU.1 could function through its transcription targets. 90 candidate genes were analyzed in this circumstance. We provided several potential candidates that could mediate the role of PU.1 in glioma through genotype-phenotype association analysis, including $B T X, F L I 1$ and $I L 1 B$. These genes were found upregulated in glioma samples in different cases. Among the potential targets of PU.1, FLII has been directly implicated with glioma cell proliferation, migration and invasion (30). Fli1 and PU.1 are two members of the ETS family of transcription factors. Previous study has reported that FLI1 expression is upregulated under transfection of SPI1 vector via binding of its promoter region (27). In glioma cells, expression of the dominant-negative form of Ets1 significantly inhibited cell proliferation and migration, it is also the case for Fli1 (30). These results implicated that other ETS family members maybe also capable of promoting glioma cell proliferation, such as PU.1.
IL1B is a cytokine that belongs to the interleukin 1 family (33). Its precursor is produced by immune cells and be cleaved by caspase- 1 to form mature IL1B (34). The cytokine is an important factor mediating immune responses, and be involved in various cellular activities, including cell proliferation, differentiation and apoptosis (35). Although the clear role of IL1B in glioma development has not been identified, previous studies have shown that immune cells in the brain can produce functional IL1B. Also, IL1B expression was altered in cells treated with conditional medium from glioma cells (36), indicating that IL1B could contribute to the progress of brain tumors. In the present study, we analyzed its gene expression level in two transcriptome databases. However, changes in IL1B expression showed opposite trends in these two datasets. It worth noting that there is a large error bar exhibited by glioblastoma group in the right panel of Fig. 6B. Nine subjects were involved in this group (the glioblastoma group in database GSE15824). Among these subjects, one showed extremely high expression of IL1B (7 fold of the average level), leading to the large error bar in this dataset. If the outlier was removed, the average expression of IL1B in glioblastoma group is $22 \%$ higher than the control group. In any case, more evidences are needed to further demonstrate whether PU.1 affect glioma through regulation of IL1B. 

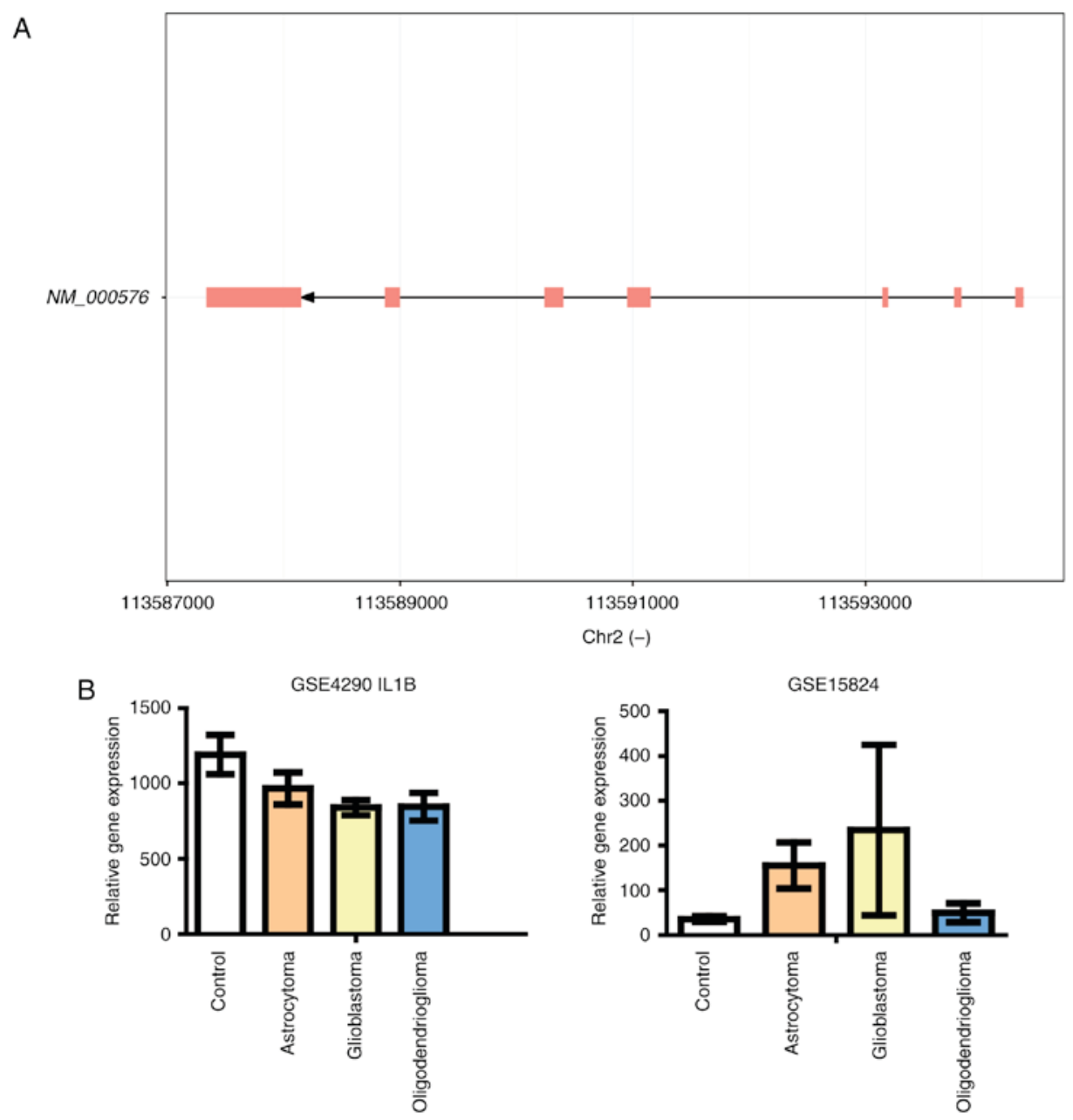

Figure 6. Relative expression of IL1B was increased in glioma patient. (A) Gene locus for IL1B transcription at the genome. Arrows, direction of transcription. Box, exons in indicated genes. (B) Fold changes of gene expression levels in three types of glioma samples. IL, interleukin.

$B T K$, a gene encoding the Bruton Tyrosine Kinase, was found to be consistently upregulated in both glioma datasets. It is one of the cytoplasmic protein tyrosine kinases that expressed by immune cells. BTK can be activated by the $\mathrm{B}$ cell receptor pathways or the $\mathrm{FcR} \gamma$ pathways (27). It has been reported that BTK activation was involved in several kinds of tumors. Although the exact role of BTK in glioma is unknown, it could be one of the important factors in such process (26).

It worth noting that the expression of these factors, such as FLI1 and IL1B, could be higher in some glioma tissues, while lower in the others. It is possible there is overexpression of an abbreviated product, through alternative splicing or post-transcriptional protein modification. Changes in the expression pattern of splicing regulator proteins were also noted at different stages of cancer progression (37). In any case, these factors could mediate the role of PU.1 in glioma. Further experimental examination is necessary to explore their function.

To explore the association between gene expression and patient prognosis, we searched TCGA database the REMBRANDT database for clinical evidence. Genomic mutations at the SPI1 locus were found in glioma patients (23). Moreover, we also noticed that patients with higher SPI1 or FLI1 (REMBRANDT database, $\mathrm{P}=0.0465$ ) expression level showed lower survival rate. These results suggest that the expression levels of some key genes could be considered as prognostic indicators of glioma malignancy.

Reactome is an online database of biological pathways $(31,32,38)$. Here we analyzed potential targets participating PU.1-associated glioma in Reactome to form a network of biological interactions in this process. Multiple pathways were identified including immune system, metabolism, gene expression and signaling transduction, indicating that PU.1 might influence the progress of glioma via different biological pathways.

In conclusion, our study provides evidence that PU.1 could play a role in the progress in glioma through its transcriptional targets. Therefore, PU.1 is involved in the regulation of glioma and probably in other types of malignant cancers through different mechanisms. These results suggest a diverse role for PU.1 in addition to its function in mediating myeloid linage differentiation and regulation of leukemia probably via its transcription targets in multiple biological pathways.

\section{Acknowledgements}

This study was supported by Cross Research Fund of Medicine and Engineering of Shanghai Jiaotong University (no. YG2016QN32) and Natural Science Foundation of Ningbo (no. 2016A610119). 


\section{References}

1. Darnell JE Jr: Transcription factors as targets for cancer therapy Nat Rev Cancer 2: 740-749, 2002.

2. Verbiest T, Bouffler S, Nutt SL and Badie C: PU.1 downregulation in murine radiation-induced acute myeloid leukaemia (AML): From molecular mechanism to human AML. Carcinogenesis 36: 413-419, 2015

3. Scott EW, Simon MC, Anastasi J and Singh H: Requirement of transcription factor PU.1 in the development of multiple hematopoietic lineages. Science 265: 1573-1577, 1994.

4. McKercher SR, Torbett BE, Anderson KL, Henkel GW, Vestal DJ, Baribault H, Klemsz M, Feeney AJ, Wu GE, Paige CJ and Maki RA: Targeted disruption of the PU.1 gene results in multiple hematopoietic abnormalities. EMBO J 15: 5647-5658, 1996.

5. Iwasaki H, Somoza C, Shigematsu H, Duprez EA, Iwasaki-Arai J, Mizuno S, Arinobu Y, Geary K, Zhang P, Dayaram T, et al: Distinctive and indispensable roles of PU.1 in maintenance of hematopoietic stem cells and their differentiation. Blood 106 1590-1600, 2005

6. Torlakovic EE, Bilalovic N, Golouh R, Zidar A and Angel S: Prognostic significance of PU.1 in follicular lymphoma. J Pathol 209: 352-359, 2006

7. Bonadies N, Pabst T and Mueller BU: Heterozygous deletion of the PU.1 locus in human AML. Blood 115: 331-334, 2010.

8. Huskova H, Korecka K, Karban J, Vargova J, Vargova K, Dusilkova N, Trneny M and Stopka T: Oncogenic microRNA-155 and its target PU.1: An integrative gene expression study in six of the most prevalent lymphomas. Int J Hematol 102: 441-450, 2015

9. Zhou J, Wu J, Li B, Liu D, Yu J, Yan X, Zheng S, Wang J, Zhang L, Zhang L, et al: PU.1 is essential for MLL leukemia partially via crosstalk with the MEIS/HOX pathway. Leukemia 28: 1436-1448, 2014.

10. de Boer J, Walf-Vorderwülbecke V and Williams O: In focus: MLL-rearranged leukemia. Leukemia 27: 1224-1228, 2013.

11. Krivtsov AV and Armstrong SA: MLL translocations, histone modifications and leukaemia stem-cell development. Nat Rev Cancer 7: 823-833, 2007

12. Toda Y, Tsukada J, Misago M, Kominato Y, Auron PE and Tanaka Y: Autocrine induction of the human pro-IL-1beta gene promoter by IL-1beta in monocytes. J Immunol 168: 1984-1991, 2002.

13. Dolecek TA, Propp JM, Stroup NE and Kruchko C: CBTRUS statistical report: Primary brain and central nervous system tumors diagnosed in the United States in 2005-2009. Neuro Oncol 14 (Suppl 5): v1-v49, 2012.

14. Wen PY and Kesari S: Malignant gliomas in adults. N Engl J Med 359: 492-507, 2008

15. Phillips HS, Kharbanda S, Chen R, Forrest WF, Soriano RH, Wu TD, Misra A, Nigro JM, Colman H, Soroceanu L, et al: Molecular subclasses of high-grade glioma predict prognosis, delineate a pattern of disease progression, and resemble stages in neurogenesis. Cancer Cell 9: 157-173, 2006.

16. Sottoriva A, Spiteri I, Piccirillo SG, Touloumis A, Collins VP, Marioni JC, Curtis C, Watts C and Tavaré S: Intratumor heterogeneity in human glioblastoma reflects cancer evolutionary dynamics. Proc Natl Acad Sci USA 110: 4009-4014, 2013.

17. Charles NA, Holland EC, Gilbertson R, Glass R and Kettenmann H: The brain tumor microenvironment. Glia 59: 1169-1180, 2011.

18. Li W and Graeber MB: The molecular profile of microglia under the influence of glioma. Neuro Oncol 14: 958-978, 2012.

19. Hu F, Ku MC, Markovic D, a Dzaye OD, Lehnardt S, Synowitz M, Wolf SA and Kettenmann H: Glioma-associated microglial MMP9 expression is upregulated by TLR2 signaling and sensitive to minocycline. Int J Cancer 135: 2569-2578, 2014

20. Pyonteck SM, Akkari L, Schuhmacher AJ, Bowman RL, Sevenich L, Quail DF, Olson OC, Quick ML, Huse JT, Teijeiro V, et al: CSF-1R inhibition alters macrophage polarization and blocks glioma progression. Nat Med 19: 1264-1272, 2013.

21. Markovic DS, Vinnakota K, van Rooijen N, Kiwit J, Synowitz M, Glass R and Kettenmann H: Minocycline reduces glioma expansion and invasion by attenuating microglial MT1-MMP expression. Brain Behav Immun 25: 624-628, 2011.
22. Yuan JX and Munson JM: Quantitative immunohistochemistry of the cellular microenvironment in patient glioblastoma resections. J Vis Exp: 2017. doi: 10.3791/56025.

23. Cancer Genome Atlas Research Network: Comprehensive genomic characterization defines human glioblastoma genes and core pathways. Nature 455: 1061-1068, 2008.

24. Poon H, Quirk C, DeZiel C and Heckerman D: Literome: PubMed-scale genomic knowledge base in the cloud. Bioinformatics 30: 2840-2842, 2014.

25. Poon H, Toutanova K and Quirk C: Distant supervision for cancer pathway extraction from text. Pac Symp Biocomput: 120-131, 2015.

26. Müller S, Sideras P, Smith CI and Xanthopoulos KG: Cell specific expression of human Bruton's agammaglobulinemia tyrosine kinase gene (Btk) is regulated by Sp1- and Spi-1/PU.1-family members. Oncogene 13: 1955-1964, 1996.

27. Starck J, Doubeikovski A, Sarrazin S, Gonnet C, Rao G, Skoultchi A, Godet J, Dusanter-Fourt I and Morle F: Spi-1/PU.1 is a positive regulator of the Fli-1 gene involved in inhibition of erythroid differentiation in friend erythroleukemic cell lines. Mol Cell Biol 19: 121-135, 1999.

28. Cinque S, Willems J, Depraetere S, Vermeire L and Joniau M: 'In vitro' effect of interleukin-1 beta on human glioma cell lines: Regulation of cell proliferation and IL-6 production. Immunol Lett 34: 267-271, 1992.

29. Kaplitt MG, Tjuvajev JG, Leib DA, Berk J, Pettigrew KD, Posner JB, Pfaff DW, Rabkin SD and Blasberg RG: Mutant herpes simplex virus induced regression of tumors growing in immunocompetent rats. J Neurooncol 19: 137-147, 1994.

30. Sahin A, Vercamer C, Kaminski A, Fuchs T, Florin A, Hahne JC, Mattot V, Pourtier-Manzanedo A, Pietsch T, Fafeur V and Wernert N: Dominant-negative inhibition of Ets 1 suppresses tumor growth, invasion and migration in rat C6 glioma cells and reveals differentially expressed Ets 1 target genes. Int J Oncol 34: 377-389, 2009.

31. Croft D, Mundo AF, Haw R, Milacic M, Weiser J, Wu G, Caudy M, Garapati P, Gillespie M, Kamdar MR, et al: The reactome pathway knowledgebase. Nucleic Acids Res 42 (Database issue): D472-D477, 2014.

32. Fabregat A, Sidiropoulos K, Garapati P, Gillespie M, Hausmann K, Haw R, Jassal B, Jupe S, Korninger F, McKay S, et al: The reactome pathway knowledgebase. Nucleic Acids Res 44: D481-D487, 2016.

33. Auron PE, Webb AC, Rosenwasser LJ, Mucci SF, Rich A, Wolff SM and Dinarello CA: Nucleotide sequence of human monocyte interleukin 1 precursor cDNA. Proc Natl Acad Sci USA 81: 7907-7911, 1984.

34. March CJ, Mosley B, Larsen A, Cerretti DP, Braedt G, Price V, Gillis S, Henney CS, Kronheim SR, Grabstein K, et al: Cloning, sequence and expression of two distinct human interleukin-1 complementary DNAs. Nature 315: 641-647, 1985.

35. Masters SL, Simon A, Aksentijevich I and Kastner DL: Horror autoinflammaticus: The molecular pathophysiology of autoinflammatory disease (*). Annu Rev Immunol 27: 621-668, 2009.

36. Zhang L, Alizadeh D, Van Handel M, Kortylewski M, Yu H and Badie B: Stat3 inhibition activates tumor macrophages and abrogates glioma growth in mice. Glia 57: 1458-1467, 2009.

37. Munkley J, Livermore K, Rajan P and Elliott DJ: RNA splicing and splicing regulator changes in prostate cancer pathology. Hum Genet 136: 1143-1154, 2017.

38. Croft D, O'Kelly G, Wu G, Haw R, Gillespie M, Matthews L, Caudy M, Garapati P, Gopinath G, Jassal B, et al: Reactome: A database of reactions, pathways and biological processes. Nucleic Acids Res 39 (Database issue): D691-D697, 2011.

This work is licensed under a Creative Commons Attribution-NonCommercial-NoDerivatives 4.0 International (CC BY-NC-ND 4.0) License. 